\title{
Perspective
}

\section{Protein dynamics elucidated by NMR technique}

\author{
Conggang Li, Chun Tang, Maili Liu \\ Key Laboratory of Magnetic Resonance in Biological Systems, Wuhan Center for Magnetic Resonance, State Key Laboratory of \\ Magnetic Resonance and Atomic and Molecular Physics, Wuhan Institute of Physics and Mathematics, Chinese Academy of \\ Sciences, Wuhan 430071, China \\ $\triangle$ Correspondence: ml.liu@wipm.ac.cn
}

In 1957, Christian Anfinsen postulated his famous dogma based on the study of ribonuclease (Sela et al., 1957), stating that the amino acid sequence of a protein determines its tertiary structure, a unique and kinetically accessible energy minimum, in a given environment. As a result, determination of a global energy minimum, or the most populated structure, has been the main focus of structural biology in the ensuing half a century. With more and more structures have been determined, people have gain some insights of why protein functions, but the detailed mechanisms and the function process, or how protein functions, remains elusive.

In recent years, mounting experimental evidences and theoretical calculations have shown that at ambient temperature, a protein rarely adopts a single native structure, but rather fluctuates among different conformations (Henzler-Wildman and Kern, 2007). The lowest-energy conformation is termed ground-state structure, conformations with higher energies and hence lower populations are termed excited-state or transient structures. The distinction between ground-state and excitedstate structures can be extended to protein-protein transient interactions, in which protein complexes have lower occurrence and hence higher energy, and the participating but uncomplexed proteins have higher occurrence and lower energies. Kinetically, the rate of inter-conversion between ground-state and transient structures is determined by the activation energy of the transition state. All together, the existence of various protein conformations and rapid exchange among them are termed protein dynamics.

It is well known that protein dynamics are crucial for protein functions, such as ligand binding, catalysis, and allosteric regulation. For example, the formation of stable protein-ligand complex is dynamical, including binding partner searching through multiple transient interactions, recognition through conformational change. Some proteins undergo subtle conformational changes, while some protein conformational changes are substantial, from one structure to another structure, or even from nonstructure to new structure. The ability of inter-conversion of different structures is indispensible for protein functions, obviously a single ground-state protein structure can't explain why/how protein exerts its functions in cells.

Although various biophysical techniques can directly or indirectly provide protein global dynamical information, nuclear magnetic resonance (NMR) is the only technique that can measure protein dynamics on pico-second to second time scale at atomic level under its functional state in solution, solid or semi-solid states. Many elegant NMR experiments, including nuclear relaxation time, heteronuclear NOE (nuclear Overhauser effect, hetNOE), relaxation dispersion experiment, RDC (Residue
Dipolar Coupling), PRE (Paramagnetic Relaxation Enhancement) and lineshape analysis, have been designed to probe protein dynamics on different time scales (Kay, 2011). Recently, an experiment shows that conformational fluctuation on ms time scale is indispensable for protein functions, knockout this dynamical fluctuations will abolish protein function while keeping the static structure intact (Bhabha et al., 2011). As there have been many reviews that have covered various NMR methods used to probe protein dynamics, in this review, we will focus on the dynamics of lowly populated states of proteins through PRE and relaxation dispersion, as well as the dynamics of membrane protein in lipid bilayer through solid-state NMR methods.

\section{PROTEIN DYNAMICS BY SOLUTION NMR}

Protein dynamics occur over a wide range of timescale, and the time scale of protein dynamics is often correlated with the length scale, e.g. picosecondnanosecond dynamics is related to side chain movement, nanosecondmicrosecond dynamics is related to loop motion, microsecond to millisecond dynamics is related to the movement of a protein domain or subunit. Importantly, protein dynamics at microsecondmillisecond are often coupled to protein functional events, such as folding, binding, allostery and catalysis. 
Unlike X-ray crystallography that investigates a protein homogenized in its crystal, NMR is uniquely suited to characterize dynamics of a protein in solution (Fig. 1). Protein dynamics at picosecond-nanosecond can be revealed through characterization of spin relaxation of ${ }^{1} \mathrm{H},{ }^{13} \mathrm{C}$ or ${ }^{15} \mathrm{~N}$, with ${ }^{15} \mathrm{~N}$ the most often. The analysis entails measurement of longitudinal relaxation rate $\left(R_{1}\right)$, transverse relaxation rate $\left(R_{2}\right)$ and hetNOE (Kay et al., 1989). hetNOE is extremely sensitive to fast time scale protein dynamics, and even becomes negative for flexible $\mathrm{N}$ - and $\mathrm{C}$ termini. The $R_{1} / R_{2}$ ratio for backbone amide indicates the orientation of $\mathrm{NH}$ bond vector relative to the overall protein anisotropic tumbling at nanosecond timescale. As a result, residues in a helix would have similar $R_{1} / R_{2}$ values, which are larger for a helix parallel to than perpendicular to the major component of the protein rotational tensor (Tang et al., 2002). The product of $R_{1} R_{2}$ indicates if the protein undergoes aggregation at increasing concentration ( $\mathrm{Li}$ and Pielak, 2009). In addition, the order parameter $S$ for protein backbone bond vector can be obtained from $R_{1}, R_{2}$ and hetNOE rates, and carries similar physical meaning to that of crystal B-factor determined at room temperature (Clore and Schwieters, 2006).

Protein chemical shift values are determined by protein conformation. When a protein undergoes exchange between two different conformations, it can be manifested by either two sets of peaks for millisecond-second timescale exchange (slow exchange), or just one set of peaks for microsecond-millisecond timescale exchange (intermediate to fast exchange). For a slow exchange, the relative intensities for the two sets of peaks can be readily measured, and the inter-conversion rate between the two can be obtained through ZZ-exchange spectroscopy or NOESY (Doucleff and Clore, 2008). For intermediate-fast exchange, relaxation dispersion, including CPMG (Mittermaier and Kay, 2006) and $R_{1 \rho}$ (Palmer and Massi, 2006) methods, have been developed to measure the contribution of chemical exchange to

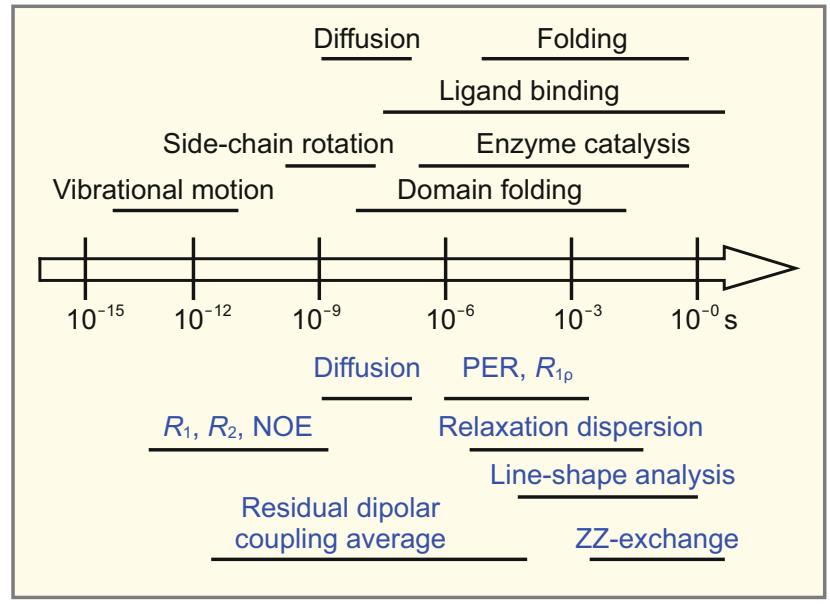

Figure 1. Major NMR methods used for probing different time scale protein dynamics.

the observed transverse relaxation rate $R_{2}$, i.e. $k_{e x}$. When assuming a two-state exchange model, the relative population of the two conformations $\left(p_{\mathrm{a}}\right.$ and $p_{\mathrm{b}}$ ), the chemical shift difference $\Delta \omega$ and the inter-conversion rate $k_{\mathrm{ex}}$ can be calculated from the $R_{\text {ex }}$.

Relaxation dispersion pinpoints residues that undergo chemical exchange at microsecond-millisecond timescale, but does not directly afford the transient conformation that the ground-state exchanges into. Recently, the structure of such transient species has been determined (Korzhnev et al., 2010; Bouvignies et al., 2011), based on the chemical shift value of the transient conformation, in conjunction with knowledge-based structure modeling software CS-Rosetta (Shen et al., 2008). However, as an indirect method, both the measurements of the transient species chemical shift and the software can cause uncertainty in the resulted structure.

As an alternative approach, protein transient structure that rapidly interconverts with the ground-state structure can be directly visualized using PRE method (Clore and Iwahara, 2009). PRE arises from through-space dipolar interactions between a paramagnetic probe attached at a specific site and nucleus under investigation. PRE is inverse proportional to the sixth power of probe-nucleus distance $r$ and is in the order of 1000 for short distances (i.e. $r<10 \AA$ ). When a protein fluctuates between ground-state and a transient structure with a shorter $r$ in the latter, the transient structure can be manifested through observed PRE, even though its population can be as small as a few percent. For exchange at lower microsecond timescale, the corresponding $k_{\text {ex }}$ is much larger than the PRE values in each conformation (Iwahara and Clore, 2006). Thus, the observed PRE can be approximated as populationweighted average for all conformational states. Excursing from the ground-state structure (which can be determined for example by X-ray crystallography), the contribution from the transient structure to overall observed PRE can be deduced. Subsequently, the transient structure can be determined through minimization of PRE energy term. Using PRE, a number of transient protein structures (Tang et al., 2007) and transient protein-protein complexes (Tang et al., 2008a, 2008b; Liu et al., 2012) have been characterized.

Also arising from dipolar interactions, FRET (fluorescence resonance energy transfer) measures distance-dependent energy transfer between two fluorophores. Though also $r^{-6}$ distance dependent, FRET measures the percentage of energy transferred from donor to acceptor and scales from 0 to 1 . FRET also depends on the relative orientation 
between two fluorophores, which is especially true for bulky fluorophores like fluorescent proteins that reorients slower than the fluorescence lifetime (Selvin, 2002). Moreover, only one distance is obtained for each FRET pair, compared to hundreds of probe-nuclei distances in a single PRE measurement. As such, FRET can in theory corroborate protein dynamics characterized by PRE, especially in the context of living cell.

For microsecond-millisecond timescale protein dynamics, the observed PRE values can be smaller than the population-weighted average of PRE values for each conformational state. Different probes possess different intrinsic paramagnetic strengths-at the same distance, $\mathrm{Mn}^{2+}$-based probe elicits PRE $\sim 5$ times stronger than spin radical does and $\sim 20$ times stronger than $\mathrm{Cu}^{2+}$ does. As a result, dynamic exchange with $k_{\mathrm{ex}}$ value comparable to PREs arising from a $\mathrm{Mn}^{2+}$ probe would be much larger than those from a $\mathrm{Cu}^{2+}$ probe attached at the same site. Thus, the observed PRE values would be closer to the population-weighted average for the $\mathrm{Cu}^{2+}$ probe, but smaller than the population-weighted average for the $\mathrm{Mn}^{2+}$ probe, and the ratio between their observed PRE values would be smaller than 20. As such, the exchange timescale can be extracted and both spatial and temporal characterization of protein dynamics can be afforded. Utilizing exchange-rate dependent scaling of observed PREs (Fig. 2), this revised version of PRE has been termed DiSPRE (Yu et al., 2009).

Despite rapid development in recent years, most of these NMR techniques assume a two-state exchange, as fitting multi-state exchange would become numerically inaccessible. Instead of fitting NMR observables, molecular dynamics (MD) simulation allows one to select conformational states from MD trajectories that would recapitulate NMR data (Long et al., 2011; Robustelli et al., 2012). Combining NMR experiments and MD simulations, future development in protein dynamics field would be to capture the transition states and fully depict transition pathways among
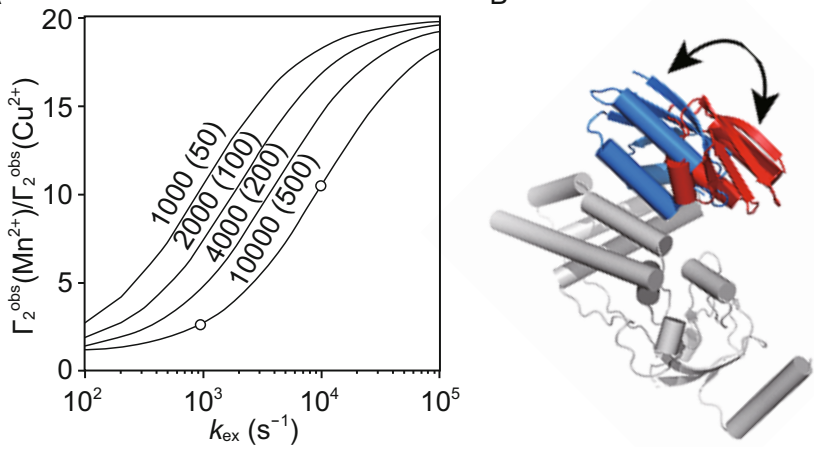

Figure 2. Proof-of-principle for differentially scaled PRE or DiSPRE. (A) For a two-state exchange with $k_{\mathrm{ex}}$ in microsecond-millisecond timescale, the ratio between observed PRE arising from $\mathrm{Mn}^{2+}$ and $\mathrm{Cu}^{2+}$ paramagnetic probes attached at the same site varies. Indicated by an open circle, if the $\mathrm{Mn}^{2+} \mathrm{PRE}$ for the minor species is 10,000 and $k_{\mathrm{ex}}=10^{4} \mathrm{~s}^{-1}$, the ratio is $\sim 10$, while the ratio is only $\sim 2$ when $k_{\mathrm{ex}}=10^{3} \mathrm{~s}^{-1}$. (B) Using DiSPRE, an alternatively binding mode of $\mathrm{HPr}$ (shown in red) that occurs at $4 \%$ of the time and exchanges with the stereospecific complex (shown in blue) at 1,100 s-1 with its partner EIN (shown in gray).

ground-state structure and various transient structures.

\section{MEMBRANE PROTEIN DYNAMICS BY SOLID-STATE NMR}

Membrane protein accounted for approximately $30 \%$ of all proteins in human genome and over $60 \%$ of the drug targets in current small drug market (Lagerstrom and Schioth, 2008). Membrane proteins serve as channels, receptors, and energy transducer and are vital to life, their malfunctions are associated with many diseases. Structure determination of membrane proteins has laid solid foundation to understand the mechanism for specificity and selectivity, but to fully understand how membrane proteins function, one static ground-state structure is not enough $(\mathrm{Hu}$ et al., 2010; Sharma et al., 2010). Membrane protein exerts its functional process is dynamical, such as the opening and close of the channel, the substance transport through the transporter. Membrane protein dynamics and its dynamical transient-state structure are urgently required to gain insights into its function (Hong, 2007; McDermott, 2009; Hong and $\mathrm{Su}, 2011$ ).

Membrane proteins differ from globu- lar proteins in that their native environment is cell membranes (Page et al., 2007). The structure, dynamics, and function of membrane proteins are influenced by the biophysical characters of this environment. Cellular membranes are composed of diverse lipid composition (>1000 different lipid species) and show transbilayer asymmetry, large chemical and electrical gradients, different dynamics and shapes. Currently used membrane mimetic environments that range from organic solvents to synthetic lipid bilayers can only model some of these properties, but not all of them. Solid-state NMR is a potential powerful tool to study membrane proteins under complex environment at very high resolution (Fu et al., 2011; Renault et al., 2012a, 2012b). Using 1D and 2D solidstate NMR of oriented sample, Diacylglycerol kinase (DAGK) from $E$. coli and the second transmembrane helices from $\mathrm{Mg}^{2+}$ transporter (CorA-TM2) were studied in different hydrophobic thickness synthetic lipid bilayers, they were found differently response to the hydrophobic mismatch, DAGK undergo very little changes while CorA-TM2 changes $10^{\circ}$ in tilt angle as the hydrophobic thickness increased (Fig. 3) (Page et al., 2007).

Unlike globular protein in aqueous 

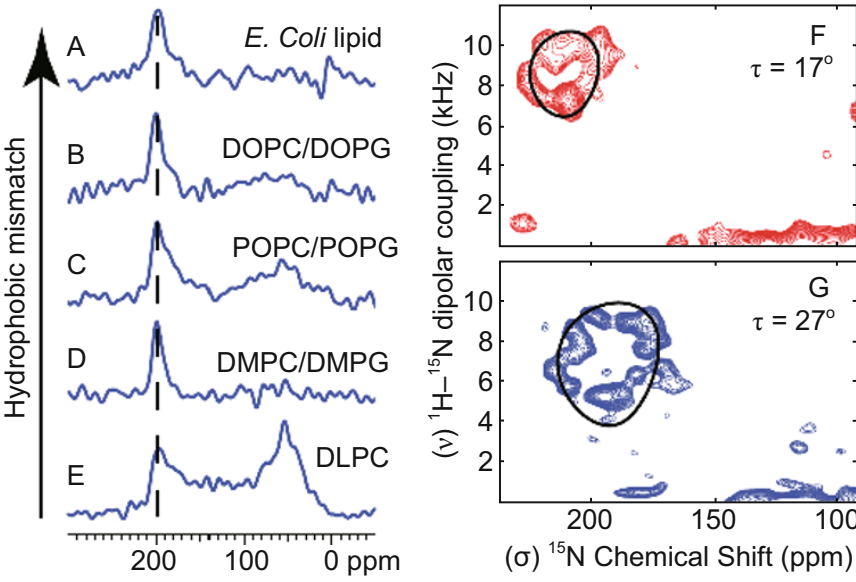

$(\sigma){ }^{15} \mathrm{~N}$ Chemical Shift (ppm)

Figure 3. The response of proteins and peptides to changes in lipid bilayer hydrophobic thickness varies depending on the types and strengths of interactions present. The location of resonances for ${ }^{15} \mathrm{~N}$-methionine labeled DAGK undergoes very little change as the hydrophobic thickness of the bilayer increases, indicating very little change in overall structure. This is shown for one-dimensional ${ }^{15} \mathrm{~N}$-spectra acquired in lipid bilayers composed of $E$. Coli lipid (A), DOPC/DOPG (B), POPC, POPG (C), DMPC/DMPG (D), and DLPC (E). Conversely, PISEMA spectra of uniformly ${ }^{15} \mathrm{~N}$-labeled CorA-TM2 indicate a $10^{\circ}$ change in helix axis tilt angle $(\tau)$ in response to an increased hydrophobic thickness for POPC/POPG lipid bilayers (F) compared to DMPC/DMPG lipid bilayers (G) (Page et al., 2007).

buffer, the global motion of membrane protein in lipid bilayer (liposome vesicle) is restrained, so the anisotropic interactions such as chemical shift anisotropy, dipolar coupling and quadrupolar interactions are not averaged out. The fast uniaxial rotational diffusion, local segmental motions and side-chain reorientation are common in membrane proteins (Shi et al., 2009; Park et al., 2011). These motions will have pronounced effects on the anisotropic interactions, the reduction of the interactions and line-shape changes from their rigid-limit values indicate motions amplitude and time scale. One-bond $\mathrm{C}-\mathrm{H}$ and $\mathrm{N}-\mathrm{H}$ dipolar couplings, ${ }^{15} \mathrm{~N},{ }^{13} \mathrm{C}$ chemical shift anisotropic and ${ }^{2} \mathrm{H},{ }^{14} \mathrm{~N}$ quadrupolar are normally employed to measure the motions mentioned above (Bertelsen et al., 2009; Chevelkov et al., 2009). 2D separated local field experiments under magic-spinning for powder sample or non-spinning for macroscopically oriented sample can resolve the anisotropic interactions of different sites in membrane proteins (Bertelsen et al., 2009; Krushelnitsky et al., 2009; Struts et al., 2011; Vostrikov et al., 2011). Relaxa- tion times can also be used to derive backbone and dynamics information of membrane proteins, but tailored labeling strategy is required in order to define the relaxation mechanism, otherwise the complicated relaxation mechanism prevents its quantitative interpretation (Schanda et al., 2010).

For water soluble globular proteins, transient structure can rapidly interconvert with the ground-state structure, but the transient structure fluctuation of membrane proteins in lipid bilayer generally occur at the slow time scale, that means NMR signals of less populated transient-state are separated from those of ground-state, we can not obtain transient-state structural information through abundant ground-state. It is also difficult to directly observe the ground-state because the transient state exist in very small percentage (less than 5\%) resulting in low sensitivity. Using chemical biology approach to lock the transient structure to enhance its population or using sensitivity enhanced method to specifically enhance the transient-state signals should be sought for transientstate structure determination. MD simu- lation combined with NMR observable parameters can also be the alternative approach to fully depict the transientstate structure and relate the structure, dynamics to its function (Vogel et al., 2010; Vostrikov et al., 2010).

\section{SUMMARY AND PERSPECTIVE}

Nowadays, more and more efforts have been devoted to studying protein function in physiological or near physiological environment, especially dynamic processes and mechanisms related to protein function (Pielak et al., 2009; Li and Liu, 2013). In this regard, NMR has an advantage over other methods due to its ability to provide information with atomic resolution in complex environment in mobile phase, closing to native state. First high-resolution 3D structure of protein in $E$. Coli has been achieved in 2009 (Sakakibara et al., 2009). But little is known about the dynamical behavior of biological macromolecules in crowded intracellular environment. The difficulties lie in the ubiquitous nonspecific interaction between proteins in cells, which impede the NMR signals observation and analysis. High concentration of polymers or proteins were added into the solution to mimic the intracellular environment, this macromolecular crowded environment was found differentially affecting translational diffusion and rotational diffusion of proteins, further quantitative studies of "crowding effects" on protein dynamics at various time scale at atomic resolution are needed (Wang et al., 2010). "Model free" approach is well established to study protein dynamics in dilute solution, but it requires that the protein is in one population (such as $100 \%$ monomer or $100 \%$ dimer) without non-specific interactions (Schurr et al., 1994), so new NMR methods other than "model free" approach for studying protein dynamics in living cells are urgently needed to develop.

In the last two decades, protein dynamical studies mainly focused on globular structural proteins, while intrinsically disordered protein and membrane proteins in lipid bilayer are rarely investigated, partly due to lacking of 
appropriate method to derive dynamical information. How to link the dynamics and function of these two types of proteins will be the future direction for protein dynamics study. This opens a new era, protein dyn-omics, for studying protein in function.

In summary, NMR plays significant roles in understanding protein functions through dynamics study, especially those functional related lowly populated high energy states, or dark state, that can not been studied by other biophysical techniques. With the rapid development of biomolecular NMR, seminal contribution of dynamical study to protein function will continue and its applications in biomedicine will be pursued in the near future.

\section{FOOTNOTES}

This work is supported by grants from the National Basic Research Program (973 Program) (Nos. 2009CB918600 and 2013СB910200), and the National Natural Science Foundation of China (Grant Nos. 21120102038 and 20921004). C.T. is a Howard Hughes Medical Insititute International Early Career Scientist.

Conggang Li declares that he has no conflict of interest. Chun Tang declares that he has no conflict of interest. Maili Liu declares that he has no conflict of interest. This article does not contain any studies with human or animal subjects performed by the any of the authors.

\section{REFERENCES}

Bertelsen, K., Paaske, B., Thogersen, L., Tajkhorshid, E., Schiott, B., et al. (2009). J Am Chem Soc 131, 18335-18342.

Bhabha, G., Lee, J., Ekiert, D.C., Gam, J., Wilson, I.A., et al. (2011). Science 332, 234-238.

Bouvignies, G., Vallurupalli, P., Hansen, D.F., Correia, B.E., Lange, O.,et al. (2011). Nature 477, 111-114.

Chevelkov, V., Fink, U., and Reif, B. (2009). J Am Chem Soc 131, 14018-14022.

Clore, G.M., and Iwahara, J. (2009). Chem Rev 109, 4108-4139.

Clore, G.M., and Schwieters, C.D. (2006). J Mol Biol 355, 879-886.
Doucleff, M., and Clore, G.M. (2008). Proc Natl Acad Sci U S A 105, 13871-13876.

Fu, R.Q., Wang, X.S., Li, C.G., SantiagoMiranda, A.N., Pielak, G.J., et al. (2011). J Am Chem Soc 133, 12370-12373.

Henzler-Wildman, K., and Kern, D. (2007). Nature 450, 964-972.

Hong, M. (2007). J Phys Chem B 111, 10340-10351.

Hong, M., and Su, Y.C. (2011). Protein Sci 20, 641-655.

$\mathrm{Hu}$, F.H., Luo, W.B., and Hong, M. (2010). Science 330, 505-508.

Iwahara, J., and Clore, G.M. (2006). Nature 440, 1227-1230.

Kay, L.E. (2011). J Magn Reson 213, 492494.

Kay, L.E., Torchia, D.A., and Bax, A. (1989). Biochemistry 28, 8972-8979.

Korzhnev, D.M., Religa, T.L., Banachewicz, W., Fersht, A.R., et al. (2010). Science 329, 1312-1316.

Krushelnitsky, A., deAzevedo, E., Linser, R., Reif, B., Saalwachter, K., et al. (2009). J Am Chem Soc 131, 12097-12099.

Lagerstrom, M.C., and Schioth, H.B. (2008). Nat Rev Drug Discov 7, 339-357.

Li, C., and Liu, M. (2013). FEBS Lett 587, 1008-1011.

Li, C., and Pielak, G.J. (2009). J Am Chem Soc 131, 1368-1369.

Liu, Z., Zhang, W.P., Xing, Q., Ren, X., Liu, M., et al. (2012). Angew Chem Int Ed Engl 51, 469-472.

Long, D., Li, D.W., Walter, K.F., Griesinger, C., and Bruschweiler, R. (2011). Biophys J 101, 910-915.

McDermott, A. (2009). Annu Rev Biophys 38, 385-403.

Mittermaier, A., and Kay, L.E. (2006). Science 312, 224-228.

Page, R.C., Li, C., Hu, J., Gao, F.P., and Cross, T.A. (2007). Magn Reson Chem 45, S2-S11.

Palmer, A.G., 3rd, and Massi, F. (2006). Chem Rev 106, 1700-1719.

Park, S.H., Casagrande, F., Das, B.B., Albrecht, L., Chu, M., et al. (2011). Biochemistry 50, 2371-2380.

Pielak, G.J., Li, C., Miklos, A.C., Schlesinger, A.P., Slade, K.M., et al. (2009). Biochemistry 48, 226-234.

Renault, M., Pawsey, S., Bos, M.P., Koers, E.J., Nand, D., et al. (2012a). Angew Chem Int Ed Engl 51, 2998-3001.
Renault, M., Tommassen-van Boxtel, R., Bos, M.P., Post, J.A., Tommassen, J., et al. (2012b). Proc Natl Acad Sci U S A 109, 4863-4868.

Robustelli, P., Stafford, K.A., and Palmer, A.G., 3rd (2012). J Am Chem Soc 134, 6365-6374.

Sakakibara, D., Sasaki, A., Ikeya, T., Hamatsu, J., Hanashima, T., et al. (2009). Nature 458, 102-105.

Schanda, P., Meier, B.H., and Ernst, M. (2010). J Am Chem Soc 132, 1595715967.

Schurr, J.M., Babcock, H.P., and Fujimoto, B.S. (1994). J Magn Reson B 105, 211224.

Sela, M., White, F.H., Jr., and Anfinsen, C.B. (1957). Science 125, 691-692.

Selvin, P.R. (2002). Annu Rev Biophys Biomol Struct 31, 275-302.

Sharma, M., Yi, M.G., Dong, H., Qin, H.J., Peterson, E., et al. (2010). Science 330, 509-512.

Shen, Y., Lange, O., Delaglio, F., Rossi, P., Aramini, J.M., et al. (2008). Proc Natl Acad Sci U S A 105, 4685-4690.

Shi, L.C., Lake, E.M.R., Ahmed, M.A.M., Brown, L.S., and Ladizhansky, V. (2009). Biochim Biophys Acta 1788, 2563-2574.

Struts, A.V., Salgado, G.F.J., and Brown, M.F. (2011). Proc Natl Acad Sci U S A 108, 8263-8268.

Tang, C., Ghirlando, R., and Clore, G.M. (2008a). J Am Chem Soc 130, 40484056.

Tang, C., Louis, J.M., Aniana, A., Suh, J.Y., and Clore, G.M. (2008b). Nature 455, 693-696.

Tang, C., Ndassa, Y., and Summers, M.F. (2002). Nat Struct Biol 9, 537-543.

Tang, C., Schwieters, C.D., and Clore, G.M. (2007). Nature 449, 1078-1082.

Vogel, A., Reuther, G., Roark, M.B., Tan, K.T., Waldmann, H., et al. (2010). Biochim Biophys Acta 1798, 275-285.

Vostrikov, V.V., Grant, C.V., Opella, S.J., and Koeppe, R.E. (2011). Biophys J 101, 2939-2947.

Vostrikov, V.V., Hall, B.A., Greathouse, D.V., Koeppe, R.E., and Sansom, M.S.P. (2010). J Am Chem Soc 132, 5803-5811.

Wang, Y., Li, C., and Pielak, G.J. (2010). J Am Chem Soc 132, 9392-9397.

Yu, D., Volkov, A.N., and Tang, C. (2009). J Am Chem Soc 131, 17291-17297. 